\title{
Electric power emergency drill evaluation based on factor analysis
}

\author{
Xiaoguang Sun*, Jianyu Geng, Kai Liu, Yuanzhi Wang and Zhipeng Wang \\ The Changchun Power Supply Company of State Grid Jilin Electric Power Company, Changchun, Jilin, China
}

\begin{abstract}
This paper explores an evaluation method to evaluate the performance of participants in electric power emergency drill, expounds various important activities in electric power emergency drill, and summarizes the general mode of emergency drill. This paper introduces the principle of factor analysis and the principal component method, then uses this method to find common factors in a series of evaluation indexes, finally takes factor score as evaluation score directly. This comprehensive evaluation method can reduce the number of evaluation indexes by looking for commonness among many indexes.
\end{abstract}

\section{Introduction}

Electric power emergency drill is an important part of electric power emergency and has irreplaceable evaluation function. On the one hand, to evaluate the power grid's ability to deal with accidents, the drill will show the power grid's technology and methods to deal with power accidents. On the other hand, for the evaluation of power grid employees' ability to deal with accidents, the drill subject is the drill personnel, and the drill performance is the best prediction of power grid employees' ability to deal with real accidents.

Starting from the general drill mode, this paper introduces the electric power emergency drill evaluation. At present, many evaluation index systems have been established. Many evaluation indexes can make the evaluation results more comprehensive, but too many indexes may lead to too much information and complicated items.

\section{Electric power emergency drill}

Literature [1] introduces the main process of emergency drill in detail. With this as a reference, the key content of electric power emergency drill is summarized, and appropriate evaluation indexes are selected.

\subsection{Key contents of the drill}

\subsubsection{News and communication.}

This part of the drill is mainly aimed at the news center, the customer service center, the science and technology information department and other relevant departments, mainly covering the release of all kinds of information.

\subsubsection{Warning information}

The source of warning information for natural disasters can be meteorological departments or historical data.
News department releases warning information in accordance with formal procedures, and warning information should contain at least the following two aspects [2].

(1) Notify the relevant personnel to enter the warning state, release warning information to the public, and take appropriate measures to try to eliminate the factors that produce warning.

(2) The operation personnel shall strengthen the operation duty, and the emergency repair personnel shall make preparations for various maintenance. In case of power grid failure, they shall quickly respond to reduce losses.

2.1.1.2 Troubleshooting information

The progress of handling accidents should be made public, including the blackout area and blackout time.

2.1.1.3 Accident summary information

Including accident causes, treatment methods and results as well as the reward and punishment information of relevant departments.

\subsubsection{Plant and station operation.}

Plant and station operation mainly includes collecting, reporting and related operations of fault information. Fault information collection covers the monitoring system background machine alarm, switch position, power flow indication, optical display plate, etc. In the whole process of accident handling, the operator of plant and station should report the accident to the dispatcher for several times, including two reports at the beginning of the occurrence of fault information (reflecting the operation status of the primary equipment and the action of the secondary equipment), and reports before and after the operation of the substation. Substation operation is the use of substation simulation system to simulate the operation of real scene.

\footnotetext{
* Corresponding author: 15601259722@163.com
} 


\subsubsection{Dispatching command.}

Dispatching command and the plant and station operation are the two most important parts of electric power emergency drill. Especially in the climax of disaster accident, dispatching is very important. Dispatching is the brain of the drill subject and issues important instructions. The content to be drilled mainly includes the following two aspects.

\subsubsection{Accident analysis}

Generally, the dispatch personnel cannot visit the fault site in person. Therefore, they need to determine the fault location and severity according to the operation report.

2.1.3.2 Output control and load transfer

In the case of the outage of main transformer substation, or even the power outage of whole station, load transfer, power pulling, power limiting and other means become extremely important. Dispatching personnel need to give instructions to adjust the operation mode of power grid according to power grid structure and power flow distribution. This part of drill is based on the dispatcher training simulation system [3].

\subsubsection{Maintenance.}

This part of drill is mainly aimed at maintenance department and material department. The fault handling of maintenance department is different from the plant and station operation, and it is not substation operation, but the replacement and maintenance of equipment parts, so it is necessary for maintenance personnel to fully understand the structure of equipment and accurately judge the damage degree of equipment, which helps to accurately estimate the maintenance time of equipment.

As the coordination department of maintenance department, the drill personnel of material department need to master various reserve information of emergency materials, including location, type, item and call time [4].

\subsection{General process of emergency drill}

The emergency drill process was set according to each stage of disaster accidents to coordinate with the specific activities of emergency drill. The general process of emergency drill is shown in Table 1.

Table 1. General process of emergency drill.

\begin{tabular}{ccc}
\hline Drill stage & Drill content & $\begin{array}{c}\text { Corresponding disaster } \\
\text { accident stage }\end{array}$ \\
\hline Preparation & $\begin{array}{c}\text { Meteorological } \\
\text { information and historical } \\
\text { data analysis } \\
\text { Warning }\end{array}$ & Produce \\
& Fault information & Development \\
collection & \\
& Failure report & \\
Accident analysis & Climax \\
Response & Substation operation & \\
& Material call and & \\
& equipment repair & \\
& Output control & \\
& Load transfer & Descending \\
Recovery & Repair report & \\
& Recovery operation & \\
& Accident evaluation & \\
\hline
\end{tabular}

\section{Factor analysis}

\subsection{Overview of factor analysis}

Principal component analysis (PCA) has been applied in many fields of electric power, and factor analysis is a kind of extension of PCA. The basic purpose of factor analysis is to describe the covariance relationship between many variables with a few random variables. These random variables are not observable and are usually called factors. In essence, the idea is to group variables according to the magnitude of correlation, so that the correlation between variables in the same group is high, and the correlation between variables in different groups is low. Due to the numerous items of evaluation indexes, although they look very detailed and comprehensive, the final conclusion should be drawn through induction and summary, which requires to explore the internal relationship between each index.

\subsection{Principle of factor analysis}

Factor analysis model assume that $\mathrm{X}$ linearly depends on a few unobservable random variables F1, F2, , , Fm (are called common factors, where $\mathrm{M}<\mathrm{P}$ ), and $\varepsilon 1, \varepsilon 2, \cdots, \varepsilon p$ are called special factors or error. Its mathematical model is:

$$
\left\{\begin{array}{l}
X_{1}-\mu_{1}=l_{11} F_{1}+l_{12} F_{2}+\mathrm{L}+l_{1 m} F_{m}+\varepsilon_{1} \\
X_{2}-\mu_{2}=l_{21} F_{1}+l_{22} F_{2}+\mathrm{L}+l_{2 m} F_{m}+\varepsilon_{2} \\
\mathrm{~L} \\
X_{p}-\mu_{p}=l_{p 1} F_{1}+l_{p 2} F_{2}+\mathrm{L}+l_{p m} F_{m}+\varepsilon_{p}
\end{array}\right.
$$

Where, $l_{i j}$ is the load of the variable $i$ on the factor $j$, which can be regarded as the effect of the common factor $j$ on the variable $i$. Unobservable random variables $F_{1}, F_{2}, \ldots$, $F_{\mathrm{m}}$ and $\varepsilon_{1}, \varepsilon_{2}, \ldots, \varepsilon_{p}$ are used to represent deviations $X_{1}-\mu_{1}$, $X_{2}-\mu_{2}, \ldots, X_{p}-\mu_{p}$.

At present, there are two most common parameter estimation methods to estimate factor load: the principal component method and the maximum likelihood method. In the principal component method, $n$ groups of data are set, and each group of data includes 6 variables, namely 6 indexes $X_{1}, X_{2}, X_{3}, X_{4}, X_{5}$ and $X_{6}$. The original matrix can be obtained. Each element in the matrix is the evaluation result collected, and $x_{i j}$ represents the value of the index $j$ in the group $i[5]$.

$$
X=\left[\begin{array}{cccccc}
x_{11} & x_{12} & x_{13} & x_{14} & x_{15} & x_{16} \\
x_{21} & x_{22} & x_{23} & x_{24} & x_{25} & x_{26} \\
\mathrm{M} & \mathrm{M} & \mathrm{M} & \mathrm{M} & \mathrm{M} & \mathrm{M} \\
x_{n 1} & x_{n 2} & x_{n 3} & x_{n 4} & x_{n 5} & x_{n 6}
\end{array}\right]
$$

The standardized data matrix is as follows:

$$
\begin{gathered}
Z=\left(z_{i j}\right)_{n} \\
z_{i j}=\left(x_{i j}-\overline{x_{j}}\right) / \sqrt{S_{j}}
\end{gathered}
$$

Among them: 


$$
\begin{gathered}
\overline{x_{j}}=\frac{1}{n} \sum_{i=1}^{n} x_{i j} \\
S_{j}=\frac{1}{n-1} \sum_{i=1}^{n}\left(x_{i j}-\overline{x_{j}}\right)^{2}
\end{gathered}
$$

Then the correlation matrix $R$ is established, and its eigenvalues and eigenvectors are calculated:

$$
R=Z^{T} Z /(N-1)
$$

Find the eigenvalues of the matrix $R: \lambda_{1} \geq \lambda_{2} \geq \ldots \geq \lambda_{6}$, and the corresponding normalized orthogonal eigenvectors can be obtained:

$$
e=\left(e_{1}, e_{2}, \mathrm{~L}, e_{6}\right)^{T}
$$

The principal component expression is:

$$
Y=Z e
$$

Calculate the contribution rate of each common factor to sample variance:

$$
\beta_{i}=\lambda_{i} / \sum_{i=1}^{6} \lambda_{i}
$$

Cumulative contribution:

$$
\beta(i)=\sum_{k=1}^{i} \lambda_{k} / \sum_{k=1}^{6} \lambda_{k}
$$

According to the principal component method, except for the constant factor $\sqrt{\lambda_{j}}$, the load on the factor $j$ is the coefficient $e_{j}$ of the population principal component $\mathrm{j}_{\mathrm{j}}$, and the factor load estimation can be obtained:

$$
l_{i j}=\sqrt{\lambda_{j} e_{i j}}
$$

Rotate the axes of the factor loads so that all points fall in the first quadrant. This is done to achieve an ideal situation where each variable has a large load on only one factor and smaller loads on the others.

\section{Application of factor analysis in electric power emergency drill}

The literature [6] list 12 indexes. Preliminary screening is carried out from these 12 indexes, and the principle of screening is: the acquisition of indexes is relatively direct and can be accompanied by the process of drill. And after the drill, the acquisition of various evaluation indexes can also be completed.

Combined with the specific assessment content, 6 indexes are selected from the 12 indexes, namely, safety production knowledge, safety operation reaction, safety operation process, safety attention, safety operation skill and safety production memory.

\subsection{Extraction of drill evaluation factors}

In the electric power emergency drill, several groups of drill evaluation data are found, then each index is similarity processed, and the factor analysis is carried out on the selected 6 indexes. Firstly, the correlation coefficient matrix of 6 variables was calculated, as shown in Table 2.
Table 2. Correlation coefficient matrix of 6 variables

\begin{tabular}{ccccccc}
\hline Variable & 1 & 2 & 3 & 4 & 5 & 6 \\
\hline 1 & 1.00 & 0.58 & 0.59 & 0.48 & 0.73 & 0.41 \\
2 & 0.58 & 1.00 & 0.42 & 0.86 & 0.41 & 0.63 \\
3 & 0.59 & 0.42 & 1.00 & 0.57 & 0.66 & 0.37 \\
4 & 0.48 & 0.86 & 0.57 & 1.00 & 0.52 & 0.65 \\
5 & 0.73 & 0.41 & 0.66 & 0.52 & 1.00 & 0.27 \\
6 & 0.41 & 0.63 & 0.37 & 0.65 & 0.27 & 1.00 \\
\hline
\end{tabular}

Note that the two data 0.86 and 0.73 , two common factors can be taken according to the factor analysis model. The eigenvalue and principal component contribution rate are shown in Table 3.

Table 3. Eigenvalue and principal component contribution rate.

\begin{tabular}{cccc}
\hline Eigenvalue & Value & $\begin{array}{c}\text { Contribution } \\
\text { rate/\% }\end{array}$ & $\begin{array}{c}\text { Cumulative } \\
\text { contribution/\% }\end{array}$ \\
\hline 1 & 3.74 & 62.28 & 62.28 \\
2 & 1.06 & 17.61 & 79.89 \\
3 & 0.46 & 7.69 & 87.58 \\
4 & 0.42 & 6.96 & 94.54 \\
5 & 0.26 & 4.38 & 98.92 \\
6 & 0.06 & 1.08 & 100.00 \\
\hline
\end{tabular}

This indicates that the contribution rate of two main components reaches $79.89 \%$, which has important practical explanation.

After factor rotation, the rotation factor load is estimated.

\subsection{Data analysis}

As can be seen from Table 4, the indexes 1, 3, 5 have a large load on $F_{2}$, which is called "professional factor" by analyzing the commonness of all indexes. The indexes 2 , 4,6 have a large load on $F_{1}$, which is called "status factor". The score of professional factor reflects the level of professional accomplishment of drill personnel, which can be improved through acquired professional training. The status factor is associated with many factors, including the physical state, work attitude, adaptability, environment of the drill personnel.

Table 4. Factor load estimation after rotation.

\begin{tabular}{cccc}
\hline \multirow{2}{*}{ Number } & Variable & \multicolumn{2}{c}{$\begin{array}{c}\text { Factor load } \\
\text { estimation after } \\
\text { rotation }\end{array}$} \\
\cline { 2 - 4 } & \multicolumn{2}{c}{ F1 } & F2 \\
\hline 1 & $\begin{array}{c}\text { Safety } \\
\text { production } \\
\text { knowledge }\end{array}$ & 0.33 & 0.81 \\
2 & $\begin{array}{c}\text { Safety operation } \\
\text { reaction }\end{array}$ & 0.87 & 0.31 \\
3 & $\begin{array}{c}\text { Safety operation } \\
\text { process }\end{array}$ & 0.28 & 0.79 \\
4 & $\begin{array}{c}\text { Safety attention } \\
\text { Safety operation } \\
\text { skill }\end{array}$ & 0.84 & 0.40 \\
6 & $\begin{array}{c}\text { Safety } \\
\text { production } \\
\text { memory }\end{array}$ & 0.16 & 0.91 \\
\hline
\end{tabular}


In the process of drill evaluation, using multiple indexes can make the evaluation mechanism more comprehensive and multi-directional. Inspired by the McClelland iceberg model, the paper tries to find a potential measurement method closer to self-concept from the surface phenomena reflected by indexes.

\subsection{Factor score based on big data technology}

In addition to the weighted least square method and the regression method, another factor score can be constructed by using a simpler method. The specific method is as follows: firstly, find out the variable with large load for each factor, and the factor score is equal to the algebraic sum of the standardized observation values of all variables with large load according to the load symbols.

This simple factor score has a high correlation with the factor score obtained by the least square method and the regression method. Although the evaluation method looks very simple, it has good summative and generalization.

\section{Conclusion}

Electric power emergency drill evaluation is the summary of emergency drill, the main content is to evaluate the emergency performance of drill personnel. By introducing the general process and key contents of the drill, the evaluation index system of the emergency drill is introduced, and some indexes in the evaluation index system are analyzed to explore their relevance and find out the common factors, so as to reduce the index dimension and keep the important information in the drill evaluation.

The evaluation methods before and after factor analysis are combined as common methods of emergency drill evaluation, so that the evaluation is comprehensive and detailed, and the key points are highlighted.

\section{Acknowledgments}

This work was financially supported by the Science and Technology Project of State Grid Jilin Electric Power Company (2020-4).

\section{References}

1. YUAN Guidong. The planning and implementation for the project of power public emergency full-scale exercise[D]. North China Electric Power University, 2009.

2. LI Yigang, YIN Guanghui, HUANG Jianfa, et al. Several key problems in emergency drill[J]. China Emergency Rescue, 2007(03): 33-35.

3. TAMURA Y, HORIE E D, et al. Development of power system simulator for research and education[J]. IEEE Trans on Power System, 1990, 5(2): 492-498.

4. HOU Hui, ZHOU Jianzhong, ZHANG Yongchuan, et al. Analysis of power emergency drills system at home and abroad and its inspiration for China[J]. Power System Protection and Control, 2010, 38(24): 236-241.

5. DAI Xinbo, CUI Yong, ZHOU Dexiang, et al. LSSVM short-term load forecasting based on principal component analysis and improved particle swarm optimization $[\mathrm{J}]$. Electrical Measurement \& Instrumentation, 2012, 49(06): 5-9.

6. DONG Yu, ZHUANG Wei, TAO Hongzhu, et al. Organization and implementation of large-scale joint anti-accident training of power system[J]. Power System Technology, 2007, 31(02): 1-4. 